\title{
An Investigation into the Use of the Impedance Cardiogram as a Predictor of Manual Chest Compression Efficacy
}

\author{
Olibhear McAlister ${ }^{1,2}$, Dewar Finlay ${ }^{1}$, Raymond R. Bond ${ }^{1}$, Daniel Guldenring ${ }^{1}$, Ben McCartney ${ }^{2}$, \\ Laura Davis $^{2}$, Hannah Torney ${ }^{1,2}$, Paul Crawford ${ }^{3}$, Frances Denny ${ }^{4}$, Rebecca Funston ${ }^{1}$, David \\ McEneaney ${ }^{5}$ \\ ${ }^{1}$ Ulster University, Newtownabbey, Belfast, UK \\ ${ }^{2}$ HeartSine Technologies Ltd., Belfast, UK \\ ${ }^{3}$ Veterinary Anaesthesia Consultant, Larne, Co Antrim, UK \\ ${ }^{4}$ Explorisitcs Ltd., Belfast, UK \\ ${ }^{5}$ Craigavon Area Hospital, Craigavon, UK
}

\begin{abstract}
High quality chest compressions (CCs) have been proven to increase patient survival, during cardiac arrest. The impedance cardiogram (ICG) may provide a simple and non-invasive CPR coaching system to improve CC efficacy of minimally trained users.

Cardiac arrest was induced in eleven swine. Manual CCs were applied at a fixed depth for two-minute episodes, increasing for each successive episode. Physiological data was recorded at 30s intervals. The primary analysis assessed the relationship between ICG amplitude and CC depth using a 5 s analysis window. The secondary analysis compared the ICG amplitude against captured physiological data and CC depth, using a 30s window.

The primary analysis yielded a strong Pearson correlation coefficient (95\% confidence interval) of 0.90 (0.89, 0.91) between ICG amplitude and CC depth. The secondary analysis produced an overall correlation of 0.92 (0.90, 0.94) between ICG amplitude and CC depth. The within-animal correlation for ICG amplitude and both end-tidal carbon dioxide and systolic blood pressure was $0.94(0.93,0.95)$ and $0.90(0.88,0.92)$ respectively.

Our data suggests that the amplitude of ICG is a good predictor of CC efficacy in a porcine model of cardiac arrest, when manual CCs are applied.
\end{abstract}

\section{Introduction}

Cardiac arrest is one of the leading causes of death in the western world. The best way to improve a patient's chance of surviving a cardiac arrest is the early onset of cardiopulmonary resuscitation (CPR) and to defibrillate the patient if needed. Current CPR guidelines, both
American Heart Association (AHA) [1] and European Resuscitation Council (ERC) [2], state that CPR delivered between a depth of $50 \mathrm{~mm}$ to $60 \mathrm{~mm}$, in an adult, provides the most efficacious therapy and maximizes the patient's chance of survival.

ERC guidelines also suggest that end tidal carbon dioxide $\left(\mathrm{ETCO}_{2}\right)$ may be used as a predictor of the return of spontaneous circulation (ROSC) and survival to hospital discharge. Patients with an $\mathrm{ETCO}_{2}$ of less than $10 \mathrm{mmHg}$ after 10 minutes of resuscitation, have been reported to have a higher mortality rate than patients with higher $\mathrm{ETCO}_{2}$ values [3], [4].

The impedance cardiogram (ICG), which is a measure of the change in impedance across a patients' thorax, has previously been used to detect cardiac output [5]. The transthoracic impedance (TTI) represents the combined total impedance, of the contents in the patient's thorax [6]. Blood flowing in and out of the thorax causes a fluctuation in the patient's TTI. The TTI signal (Z) may be acquired by passing a low AC current $(50 \mu \mathrm{A})$ with a high frequency $(32 \mathrm{kHz})$ through the thorax. The ICG signal $(\Delta \mathrm{Z})$ is obtained by applying a high pass or band pass filter to the TTI signal [6]-[8].

The ICG has previously been shown to be a good predictor of chest compression (CC) depth, when mechanical $\mathrm{CCs}$ are delivered to a porcine model of cardiac arrest, via a pneumatic piston [9]. It has also been reported that ICG and TTI may be utilized to develop highly accurate CPR rate feedback algorithms [10], [11].

This study was conducted to primarily assess the relationship between ICG amplitude and CC depth. The relationship between ICG amplitude and other endpoints including $\mathrm{ETCO}_{2}$, systolic blood pressure (SBP), diastolic blood pressure (DBP) and pulse oximetry $\left(\mathrm{SpO}_{2}\right)$, during manual cardiac massage were also assessed. 


\section{Materials and Methods}

\subsection{Data Collection}

Data was collected from an eleven-model porcine study, conducted between July 2016 and August 2016. Electrocardiogram (ECG) and ICG signals were recorded via a HeartSine Technologies defibrillator. The ECG and ICG signals were sampled at a frequency of $250 \mathrm{~Hz}$ with a resolution of $0.154 \mu \mathrm{V}$ per least significant bit (LSB) and $0.315 \mathrm{~m} \Omega / \mathrm{LSB}$ respectively. CC depth was measured using a Phillips HeartStart MRx with Q-CPR technology. The CC depth measurements were sampled at a frequency of $50 \mathrm{~Hz}$, with a resolution of $0.01 \mathrm{~mm} / \mathrm{LSB}$.

Cardiac arrest was induced in each animal at the beginning of the study protocol. Chest compressions were applied to each animal in the form of two-minute episodes. Each episode consisted of continuous CCs delivered at a rate between 100-120 compressions per minute (CPM), at a maintained depth. A total of seven episodes of CPR were delivered to each animal. The first four episodes of CPR for each animal were delivered at a depth between $10 \mathrm{~mm}$ and $39 \mathrm{~mm}$ and the last three CPR episodes were applied at depth between $40 \mathrm{~mm}$ and $60 \mathrm{~mm}$.

In addition to the acquired ICG and compression depth signal, vital sign measurements were recorded. $\mathrm{ETCO}_{2}$, $\mathrm{SpO}_{2}, \mathrm{SBP}$ and DBP measurements, were transcribed from a Datex-Ohmeda vital signs monitor.

\subsection{Methods}

Upon completion of data collection, the ICG and CC depth signals were subject to annotation review by a trained study investigator. This was conducted to synchronize the ICG and CC depth signals, as they were obtained using different devices with no real-time clock to automatically align the output signals. The annotation process involved plotting the ICG and CC depth signals and identifying a common event, such as the beginning of a two-minute CPR episode. The sample index at which the common event occurred was recorded for each signal and converted to relative time. The duration between the two recorded times was calculated and used to trim the beginning of the signal with the greater time to event. A second annotation review was conducted to identify the start time of each CPR episode using the ICG signal. The second round of annotations were used to segment the ICG and CC depth signal data into two-minute epochs representing individual chest compression episodes.

Once the signals were synchronized and segmented, into individual episodes, they were ready for processing and analysis. The primary analysis used a non-overlapping 5 second window to obtain data pairs of ICG amplitude and CC depth amplitudes and the relationship between these variables was evaluated. The secondary analysis implemented a 30 second non-overlapping analysis window with ICG and CC depth data pairs incorporated into the table of transcribed vital signs and $\mathrm{ETCO}_{2}$ data.

\subsubsection{Signal Analysis}

ICG amplitude was represented by calculating the root mean squared (RMS) of the ICG signal for each analysis window. CC depth was calculated using a custom made, differentiation based, peak detection algorithm. The peak detection algorithm was responsible for identifying samples at which local minima occurred in the CC depth signal. To avoid the identification of false peaks, a rule was introduced that peaks should be at least 25 samples apart, as CPR was administered between $100 \mathrm{CPM}$ and $120 \mathrm{CPM}$ during the study. Each observed minima is representative of the maximal sternum displacement for a single CC. The mean of the identified minima, within each analysis window, was used to represent $\mathrm{CC}$ depth for statistical analysis. Figure 1 shows the identification of local minima within the CC depth signal.

The ICG amplitude and CC depth processing methods were applied to the signal data twice, once with a 5 second analysis window for the primary analysis and again with a 30 second analysis window for the secondary analysis. Data pairs obtained from the 5 second window analysis were compiled for correlation analysis. Data pairs for the 30 second window analysis were compiled and added to a table of transcribed vital sign and $\mathrm{ETCO}_{2}$ data.

As model residuals were not normally distributed within the vital signs and $\mathrm{ETCO}_{2}$ transcriptions, all variables for the secondary analysis were replaced with their corresponding ranks prior to statistical analysis. Both within-animal and overall correlations were calculated for ICG amplitude and CC depth. Within-animal correlations were calculated for ICG amplitude and each recorded vital sign and $\mathrm{ETCO}_{2}$.

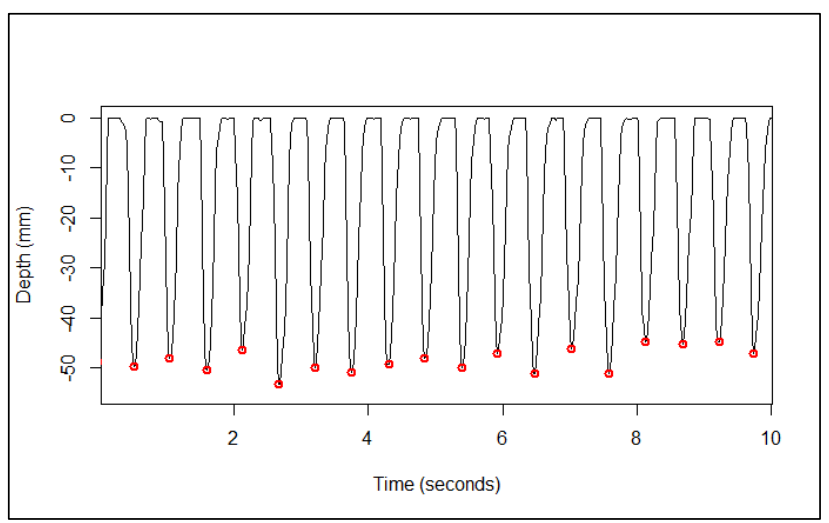

Figure 1 Example of a CC depth trace displaying the identification of local maxima 


\section{Results}

\subsection{Primary Analysis}

For the primary analysis, a total of 1848 (24 analysis windows $x 7$ CPR episodes $x 11$ animals $=1848$ data pairs) data pairs of ICG amplitude and CC depth were available for statistical analysis. A strong Pearson correlation coefficient (95\% confidence interval) of $0.90(0.89,0.91)$ was observed between depth of chest compression and ICG amplitude. The within-animal correlation was 0.94 $(0.92,0.96)$. The distribution of chest compression depth and ICG amplitudes are shown in Figure 2.

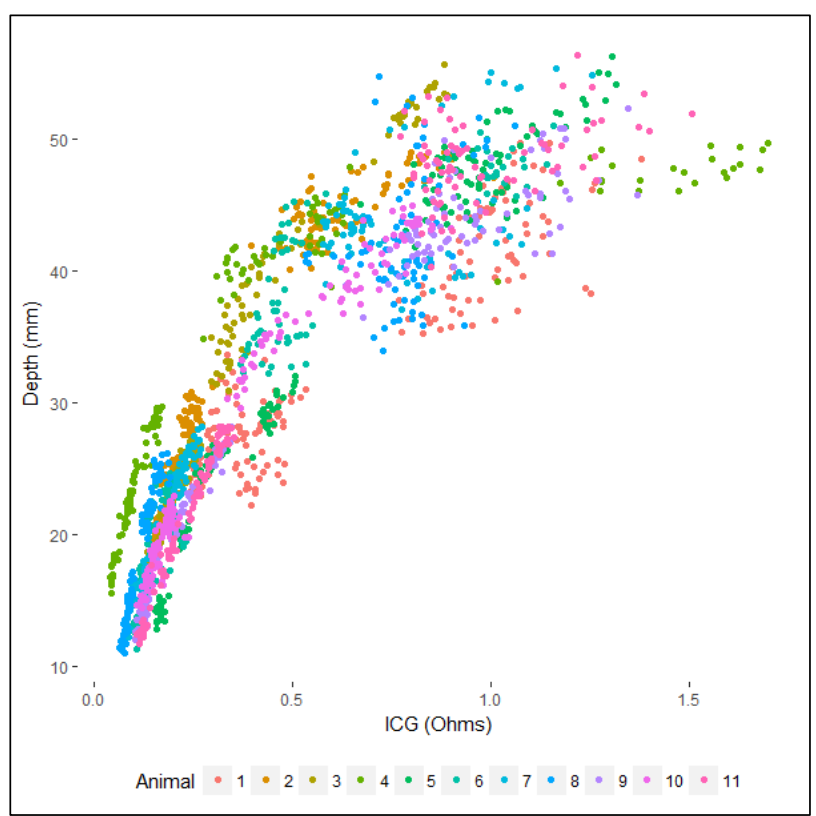

Figure 2 Relationship between ICG amplitude and CC depth using a 5 second analysis window

\subsection{Secondary Analysis}

A total of 308 (4 analysis windows $x 7$ CPR episodes $\mathrm{x}$ 11 animals $=308$ observations) observations were collected for all variables except for $\mathrm{SpO}_{2}$ which produced only 285 observations. The overall correlation (95\% confidence interval) between ICG amplitude and CC depth was $0.92(0.90,0.94)$. The within-animal correlation between ICG amplitude and CC depth yielded a correlation estimate of $0.96(0.95,0.97)$. The within-animal correlation estimate for ICG amplitude and $\mathrm{ETCO}_{2}$ was $0.94(0.93,0.95)$. All within-animal correlations can be seen in Table 1.
Table 1 Observed correlation estimates between ICG amplitude and recorded endpoints $\left(\mathrm{CC}\right.$ depth, $\mathrm{ETCO}_{2}, \mathrm{SBP}, \mathrm{DBP}$ and $\mathrm{SpO}_{2}$ ), using a 30 second analysis window.

\begin{tabular}{llll}
\hline Endpoint & $\mathrm{N}$ & Corr. & $95 \% \mathrm{Cl}$ \\
\hline CC Depth & 308 & 0.96 & $(0.95,0.97)$ \\
$\mathrm{ETCO}_{2}$ & 308 & 0.94 & $(0.93,0.95)$ \\
$\mathrm{SBP}$ & 308 & 0.90 & $(0.88,0.92)$ \\
$\mathrm{DBP}$ & 308 & -0.64 & $(-0.71,-0.55)$ \\
$\mathrm{SpO}_{2}$ & 285 & -0.03 & $(-0.14,0.10)$ \\
\hline
\end{tabular}

\section{Discussion}

The results from the primary analysis identifies that there is a strong relationship $(\mathrm{r}=0.94)$ between ICG amplitude and chest compression depth. Due to the short 5 second analysis windows and the monotonicity of the dataset, the primary analysis demonstrates it is possible to rapidly process the ICG signal and subsequently, provide CPR performance feedback to the user.

The strong observed correlations between ICG amplitude and physiological parameters such as $\mathrm{ETCO}_{2}$ $(r=0.94)$ and SBP $(r=0.90)$, provides a solid framework for developing an ICG based CPR feedback system. Quantifying the physiological response of a patient, as CPR is performed, is a preferred indicator of efficacy. Whilst high CC depth is associated with higher blood pressure and circulation during resuscitation efforts, the guideline depths of $50 \mathrm{~mm}$ to $60 \mathrm{~mm}$ have been proven to be unattainable, even with real time feedback [12]. An argument can be made that CC depth is a strictly mechanical measurement of sternum displacement and is not achievable on all patients. The application of $50 \mathrm{~mm}$ to $60 \mathrm{~mm}$ CCs, regardless of patient build, may not yield the best survival outcome. The utilization of ICG as a direct measure of hemodynamic flow during CPR, has the potential to provide the optimal CC depth tailored for each individual patient.

\section{Limitations}

The study was conducted in a well-controlled laboratory environment which reduced the likelihood of external interference. All animals enrolled in the study were healthy and of similar age. The weight of the enrolled animals was restricted, with an observed range of $33.0 \mathrm{Kg}-43.5 \mathrm{Kg}$. Electrode placement was standardized during the investigation and a narrow TTI range across all animals of $39.18 \Omega$ to $59.38 \Omega$ was observed, indicating good electrode adhesion.

Manual CPR was applied to each animal by a single veterinarian which minimized variance in $\mathrm{CPR}$ hand positioning. An additional limitation of this research was that CC depths of $50 \mathrm{~mm}$ and greater were difficult to 
achieve.

\section{Conclusions}

The demand for a simple intuitive CPR feedback system has never been greater. The installation of AEDs in public spaces, work environments and leisure facilities has become a common occurrence. Early defibrillation is imperative in the treatment of SCA, however, without effective CPR, the chance of survival drops rapidly.

Developing an intuitive CPR feedback system, which minimally trained users can operate, may increase CPR efficacy, thus, improving the patients chance of survival. Reducing the number of peripheral accessories required to coach the user on CPR, as well as, delivering defibrillation therapy, would improve the usability of such a system [13].

The high overall correlation between ICG amplitude and $\mathrm{CC}$ depth for both the primary and secondary analysis $\left(r_{\text {primary }}=0.90, r_{\text {secondary }}=0.92\right)$, indicates that ICG is a good predictor of CC depth during CPR. The ICG may be used to develop a non-perplexing CPR coaching tool which adheres to the current AHA and ERC resuscitation guidelines.

\section{Conflicts of interest}

OMcA, BMcC, LD, HT and RF are employed by HeartSine Technologies. OMcA and HT are part-time PhD students at Ulster University. DMcE is a member of HeartSine Technologies clinical advisory board. This research was funded by HeartSine Technologies.

\section{References}

[1] M. E. Kleinman et al., "Part 5: Adult basic life support and cardiopulmonary resuscitation quality: 2015 American Heart Association guidelines update for cardiopulmonary resuscitation and emergency cardiovascular care," Circulation, vol. 132, no. 18, pp. S414-S435, 2015.

[2] K. G. Monsieurs et al., "European Resuscitation Council Guidelines for Resuscitation 2015. Section 1. Executive summary," Resuscitation, vol. 95, pp. 1-80, 2015.

[3] C. W. Callaway et al., Part 4: Advanced life support: 2015 International consensus on cardiopulmonary resuscitation and emergency cardiovascular care science with treatment recommendations, vol. 132. 2015.
[4] J. Soar et al., "European Resuscitation Council Guidelines for Resuscitation 2015. Section 3. Adult advanced life support.," Resuscitation, vol. 95, pp. 100 147, 2015.

[5] M. G. Schultz, R. E. D. Climie, S. B. Nikolic, K. D. Ahuja, and J. E. Sharman, "Reproducibility of cardiac output derived by impedance cardiography during postural changes and exercise," Artery Res., vol. 6, no. 2, pp. 78-84, 2012.

[6] T. Sodolski and A. Kutarski, "Impedance cardiography : A valuable method of evaluating haemodynamic parameters," vol. 14, no. 2, pp. 115-126, 2007.

[7] P. W. Johnston, Z. Imam, G. Dempsey, J. Anderson, and A. A. J. Adgey, "The transthoracic impedance cardiogram is a potential haemodynamic sensor for an automated external defibrillator," Eur. Heart J., vol. 19, no. 12 , pp. 1879-1888, 1998.

[8] Z. Lababidi, D. A. Ehmke, R. E. Durnin, P. E. Leaverton, and R. M. Lauer, "The First Derivative Thoracic Impedance Cardiogram," Circulation, vol. 41, no. 4. pp. 651-658, 1970.

[9] A. Howe et al., "An investigation of thrust, depth and the impedance cardiogram as measures of cardiopulmonary resuscitation efficacy in a porcine model of cardiac arrest," Resuscitation, vol. 96, pp. 114 $120,2015$.

[10] L. Wik, J. K. Russell, J. Kramer-johansen, T. Eftestøl, and E. Alonso, "Chest compression rate feedback based on transthoracic impedance," Resuscitation, vol. 93, pp. 82-88, 2015.

[11] H. Kwok, J. Coult, C. Liu, and J. Blackwood, “An accurate method for real-time chest compression detection from the impedance signal," Resuscitation, vol. 105, pp. 22-28, 2016.

[12] T. G. Kampmeier et al., "Chest compression depth after change in CPR guidelines-Improved but not sufficient," Resuscitation, vol. 85, no. 4, pp. 503-508, 2014.

[13] H. Torney et al., "A Usability Study of a Critical Man Machine Interface : Can Layperson Responders Perform Optimal Compression Rates When Using a Public Access Defibrillator with Automated Real-Time Feedback During Cardiopulmonary Resuscitation?," IEEE Trans. Human-Machine Syst., vol. 46, no. 5, pp. 749-754, 2016.

Address for correspondence.

Olibhéar McAlister

Ulster University, NIBEC, Newtownabbey BT37 OQB mcalister-o2@ulster.ac.uk 